\title{
Teaching with data: Engaging students in learning through the use of authentic data
}

\begin{abstract}
ADINA PAYTAN1
1University of California Santa Cruz

Data are at the heart of what we do as scientists, data are becoming increasingly important in science and society, and thus data literacy is a vital asset to students as they prepare for careers in and outside science, technology, engineering, and mathematics and go on to lead productive lives. Short of engaging students directly in data collection, evaluation and interpretation, in today's knowledge driven world in which access to information is no longer a barrier, educators have unprecedented opportunities to introduce learners in authentic data. Engaging students directly with authentic data provides an opportunity to model the scientific process through inquiry and active learning, allows students to engage in real-world problems, scenarios, and challenges and enables them to learn important skills and content. Working with authentic data can simultaneously teach the methods of a data-based approach while also illuminating a particular topic. Using authentic geoscience data in courses can help students develop geoscientific analytical and reasoning skills, improve depth of understanding, enhance retention of content, and build skills useful across a wide range of professions. Working hands-on with real data is also key to building student appreciation for the strengths and weakness of scientific inquiry as a way of knowing. A foundation that is especially critical when tackling the controversies that arise around societal challenges. In this session we will examine the skills used in working with authentic data and explore a framework for fostering data literacy. General guidelines and examples of lesson plans and resources will be discussed.
\end{abstract}

